\title{
Study on the Ecological Situation and the Nursery Function of the Posidonia oceanica (L.) Delile, 1813 Prairies on the Islands of Ventotene e Santo Stefano
}

\author{
Roberto Bedini $^{1^{*}}$, Marco Bedini ${ }^{1}$, Arianna Trafeli $^{1}$ and Martina Manuele ${ }^{1}$ \\ ${ }^{1}$ Istituto di Biologia ed Ecologia Marina, Piazza Bovio n. 3/4, C.A.P. 57025, Piombino (LI) Italy.
}

Authors' contributions

This work was carried out in collaboration among all authors. All authors read and approved the final manuscript.

Article Information

DOI: 10.9734/ARRB/2021/v36i730399

Editor(s):

(1) Dr. Bechan Sharma, University of Allahabad, India.

Reviewers:

(1) Suneetha V, Vellore Institute of Technology, India.

(2) Jinqing Wang, Weifang University, China. Complete Peer review History: https://www.sdiarticle4.com/review-history/70769

Original Research Article

Received 02 May 2021

Accepted 07 July 2021

Published 24 July 2021

\section{ABSTRACT}

On behalf of the Management of the Protected Marine Area of the island of Ventotene and Santo Stefano the Marine Ecology and Biology Institute of Piombino (ITALY) carried out a very in dept study aimed at knowledge of the ecological situation of the Posidonia Oceanica (L.) Delile, 1813 meadows. The most appropriate methodologies were used to be able to identify all the benthic species present in order to evaluate the efficiency of the nursery function of the prairies investigated. The results of the numerous species captured in the juvenile stage have demonstrated the excellent nursery function of the meadows of the island of Ventotene.

Keywords: Nursery; biocenoses; Marine Protected Area.

\section{INTRODUCTION}

After carring out a study on the vagile biocenoses an in-depth study was carried out on the ecological situation and on the very important nursery function of the Posidonia oceanica (L.)
Delile, 1813 prairies in the Marine Protected Area of the islands of Ventotene and Santo Stefano (Fig. 1).

All the methods were used, both in immersion and in the laboratory, suitable for identify all the 
vagile and sessile species present on the leaves and in the prairie.

\section{MATERIAL AND METHODS EXPERIMENTAL METHODOLOGY \\ DETAILS}

The study was performed in three zones of the AMP $(a, b, c)$ at two bathymetric intervals $(4-7 \mathrm{~m}$ and 12-18 m) (Fig. 1 and Table 1).

The methods used are the following:

\subsection{Visual Census}

Diving through a transept of $25 \mathrm{~m}$ along the bathymetric profile (Figs. 2 and 3) [1] conspicuous sessile and floating species have been identified along the bathymetric profile by noting and phothogeaphyng the observed species and their abundance [2,3,4]. 4 replicates were performed for each bathymetric interval.

\subsection{Sampling with a Plankton Net}

To evaluate the nursery function $[5,6,7,8,9,10,11]$, a diving biologist with a plankton net (with $1 \mathrm{~mm}$ mesh) captures juvenile species, in reproduction species present on and between the $P$. oceanica leaves (Fig. 4) that have been taxonomically defined [12, 13, 14]. 3 replicates were performed for each bathymetric interval (Fig. 5).

\subsection{Bunch Counting}

To evaluate the leaves density in each prairie a diving biologist used a $30 \times 30 \mathrm{~cm}$ square for counting the $P$. oceanica bunches (Fig. 6 ). After that the density of the bunches was calculated $x$ $\mathrm{m}^{2}[15,16,17]$.

\section{RESULTS}

\subsection{Bunch Counting (3 replicates)}

The count of the bunches indicate a very good situation in the prairies investigated which show a very particular conformation given by a continuous presence of boulders in the prairies.

\subsection{Visual Census}

\section{Sampling with a Plankton Net}

The study was not possible to carry out in the bathymetric interval $0-3 \mathrm{~m}$ as far as this depth is no $P$. oceanica in any of the studied areas.

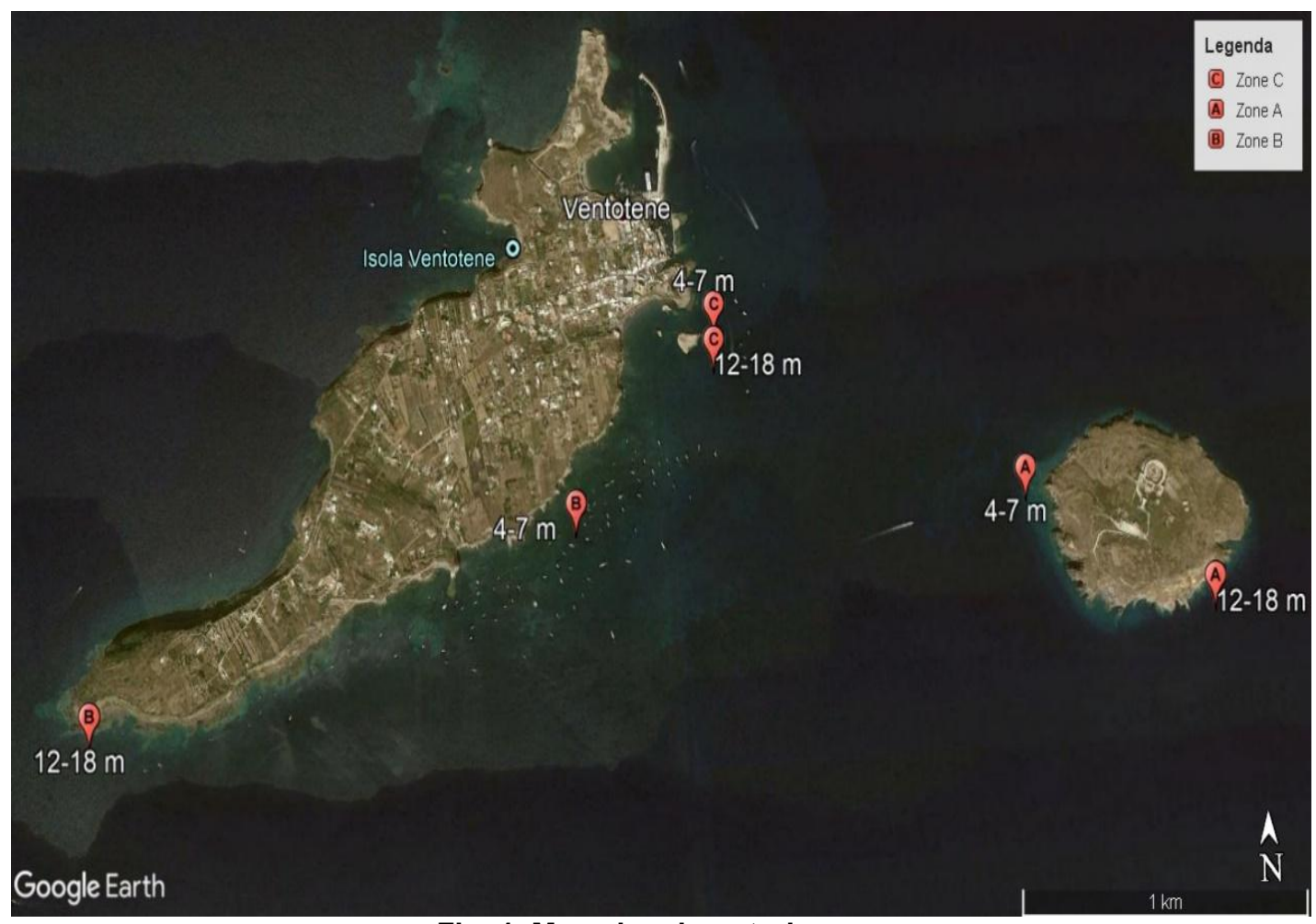

Fig. 1. Map showing study area 
Table 1. Coordinates of study area

\begin{tabular}{lll}
\hline $\begin{array}{l}\text { Zone of the Marine Protected } \\
\text { Area }\end{array}$ & Bathymetric interval $(\mathbf{m})$ & $\begin{array}{l}\text { WGS84 coordinates of the } \\
\text { survey sites }\end{array}$ \\
\hline Zone A & $4-7$ & $40^{\circ} 47^{\prime} 24.7^{\prime \prime} \mathrm{N} 13^{\circ} 26^{\prime} 57.1^{\prime \prime} \mathrm{E}$ \\
& $12-18$ & $40^{\circ} 47^{\prime} 13.8^{\prime \prime} \mathrm{N} 13^{\circ} 27^{\prime} 25.2^{\prime \prime} \mathrm{E}$ \\
\hline Zone B & $4-7$ & $40^{\circ} 47^{\prime} 22.9^{\prime \prime} \mathrm{N} 13^{\circ} 25^{\prime} 47.1^{\prime \prime} \mathrm{E}$ \\
& $12-18$ & $40^{\circ} 47^{\prime} 04.8^{\prime \prime} \mathrm{N} 13^{\circ} 24^{\prime} 32.9^{\prime \prime} \mathrm{E}$ \\
\hline Zone C & $4-7$ & $40^{\circ} 47^{\prime} 41.8^{\prime \prime} \mathrm{N} 13^{\circ} 26^{\prime} 9.38^{\prime \prime} \mathrm{E}$ \\
& $12-18$ & $40^{\circ} 47^{\prime} 38.3^{\prime \prime} \mathrm{N} 13^{\circ} 26^{\prime} 09.2^{\prime \prime} \mathrm{E}$ \\
\hline
\end{tabular}

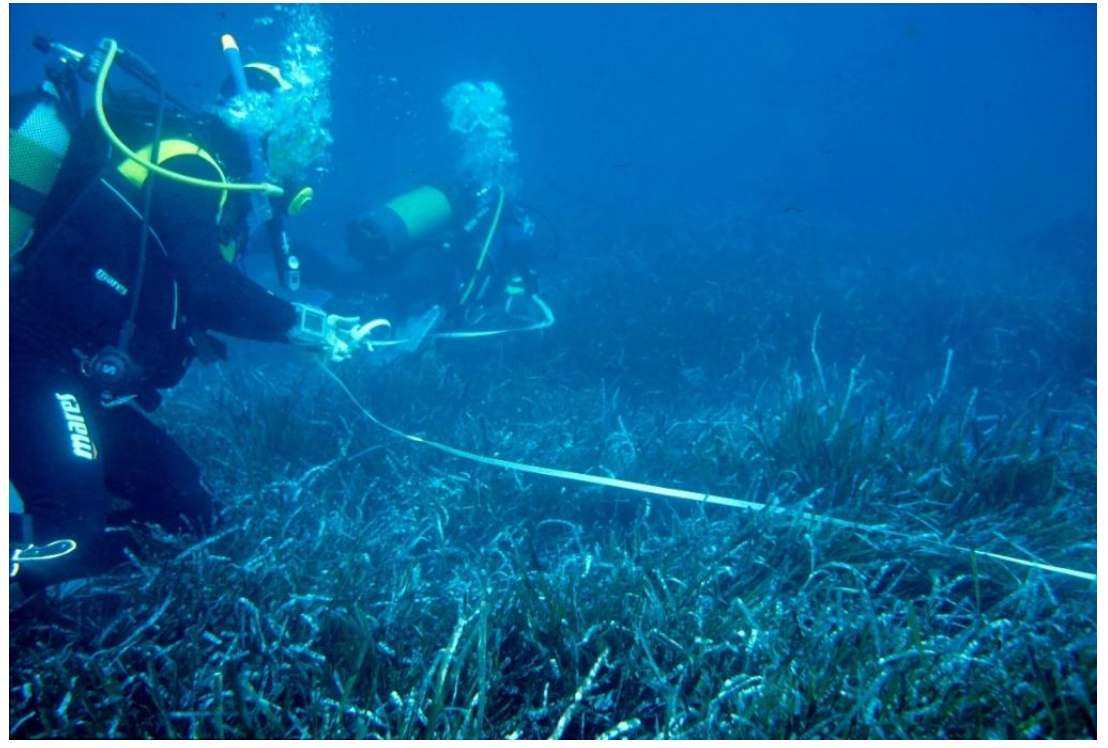

Fig. 2. Transept placement

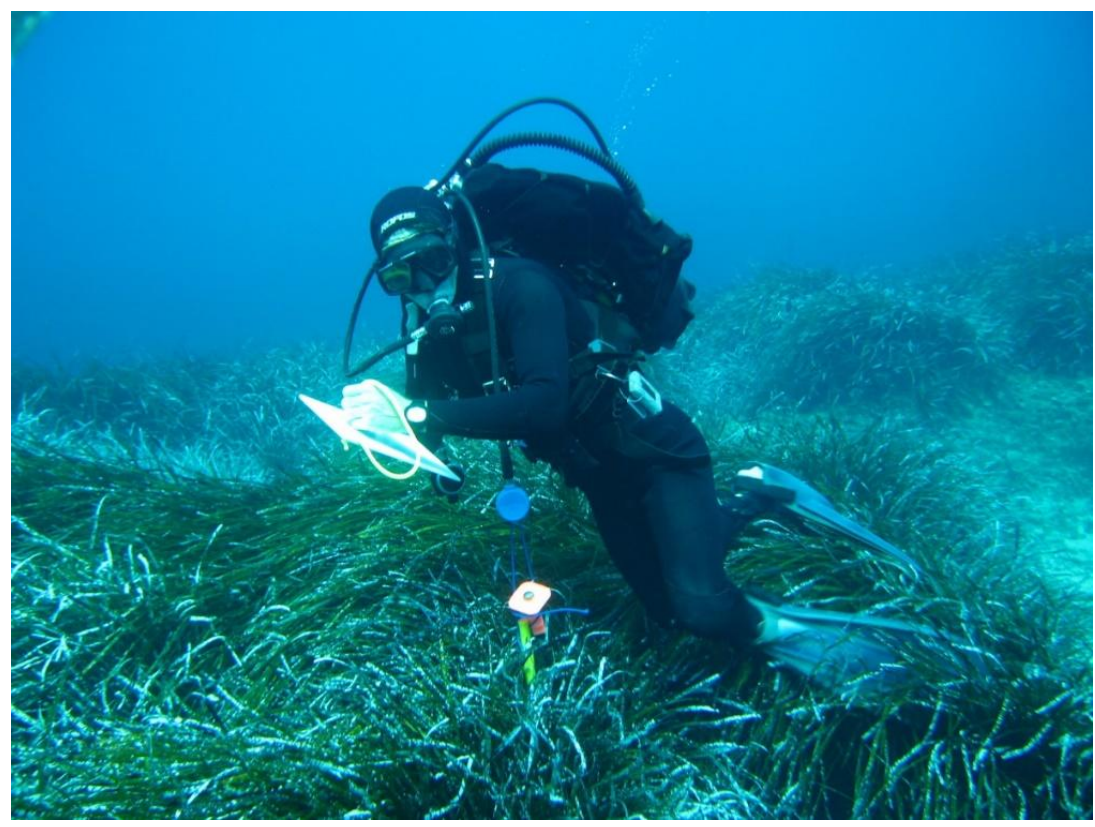

Fig. 3. Visual census 


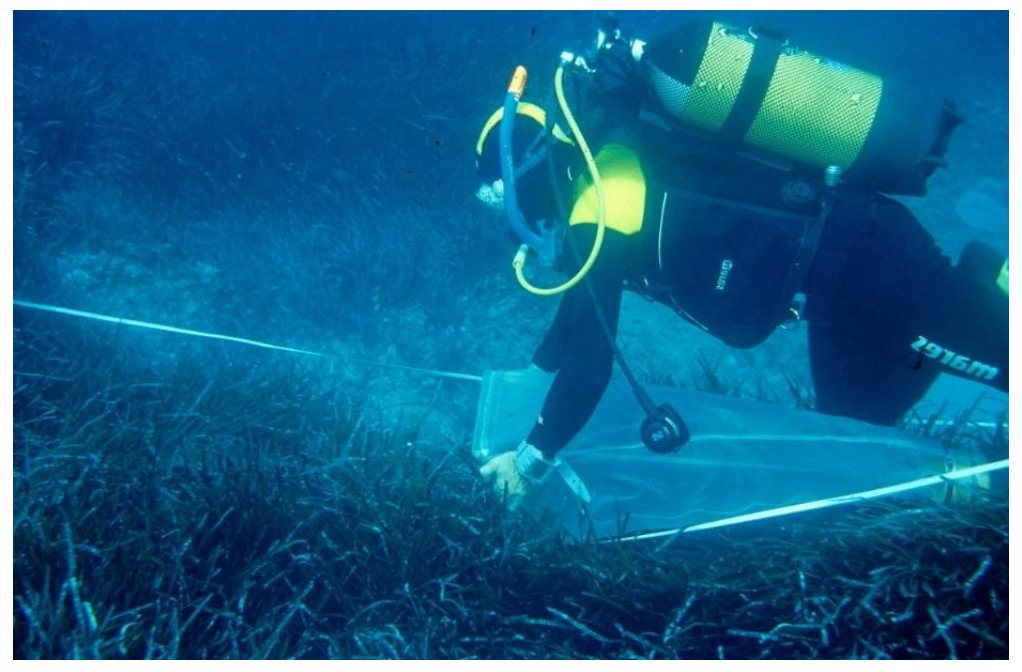

Fig. 4. captures juvenile species from $P$. oceanica leaves

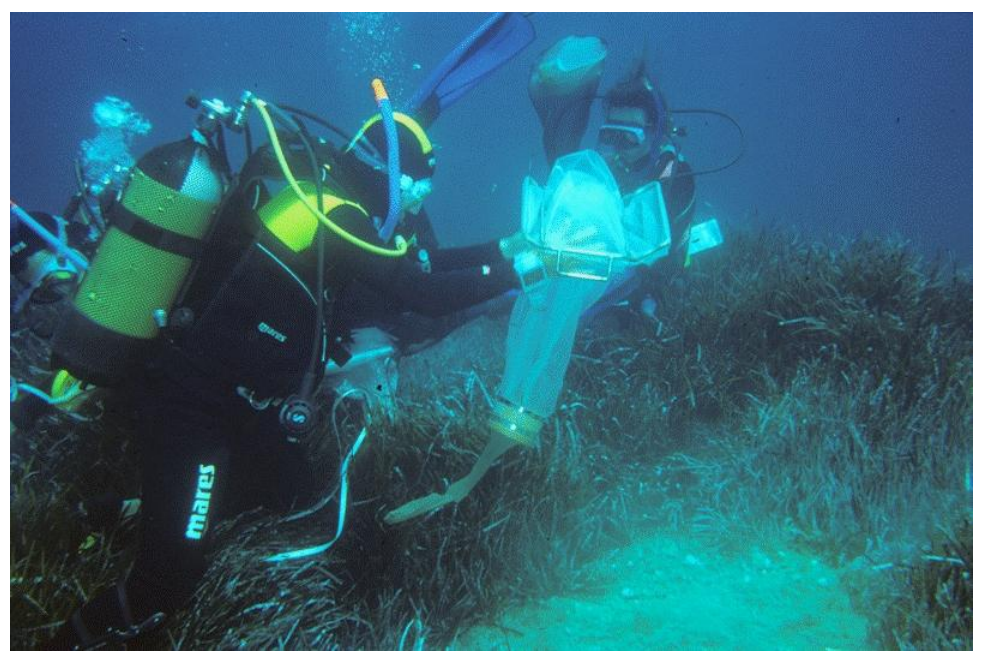

Fig. 5. Sampling

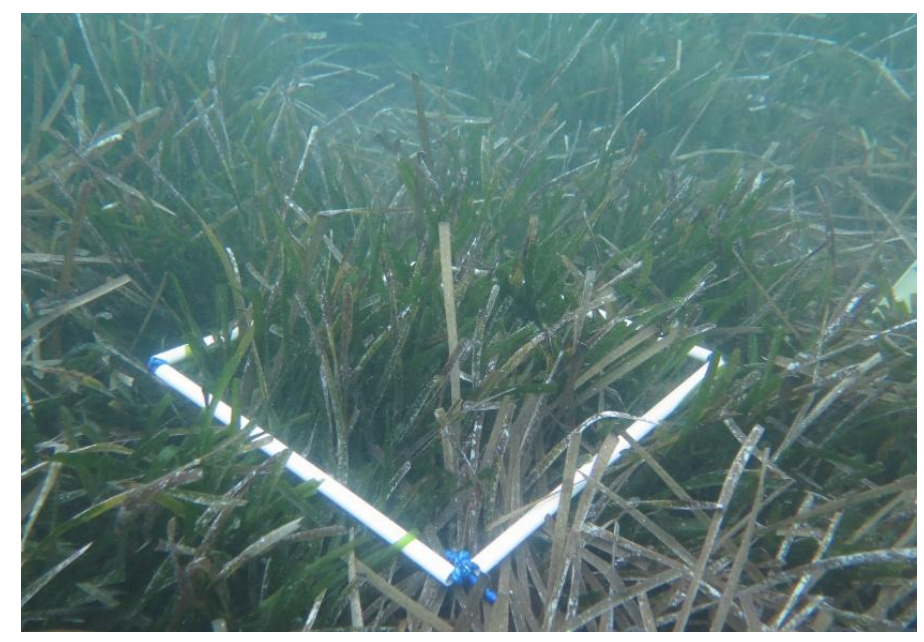

Fig. 6. Bunch counting 


\begin{tabular}{|c|c|c|}
\hline Area $A$ at the bathymetric interval $4-7 \mathrm{~m}$ & $\begin{array}{l}\text { Bunch number } \times \mathrm{m}^{2}=800 ; 744 ; \\
700\end{array}$ & Very dense prairie \\
\hline Area $B$ at the bathymetric interval $4-7 \mathrm{~m}$ & $\begin{array}{l}\text { Bunch number } \times \mathrm{m}^{2}=511 ; 600 \text {; } \\
622\end{array}$ & Dense prairie \\
\hline Area $\mathrm{C}$ at the bathymetric interval $4-7 \mathrm{~m}$ & $\begin{array}{l}\text { Bunch number } \times \mathrm{m}^{2}=822 ; 767 \text {; } \\
789\end{array}$ & Very dense prairie \\
\hline $\begin{array}{l}\text { Area } A \text { at the bathymetric interval } 12- \\
18 \mathrm{~m}\end{array}$ & $\begin{array}{l}\text { Bunch number } \times \mathrm{m}^{2}=822 ; 844 \text {; } \\
756\end{array}$ & Very dense prairie \\
\hline $\begin{array}{l}\text { Area } B \text { at the bathymetric interval } 12- \\
18 \mathrm{~m}\end{array}$ & $\begin{array}{l}\text { Bunch number } \times \mathrm{m}^{2}=778 ; 711 \text {; } \\
678\end{array}$ & Very dense prairie \\
\hline $\begin{array}{l}\text { Area } \mathrm{C} \text { at the bathymetric interval 12- } \\
18 \mathrm{~m}\end{array}$ & $\begin{array}{l}\text { Bunch number } \times \mathrm{m}^{2}=844 ; 733 \text {; } \\
800\end{array}$ & Very dense prairie \\
\hline
\end{tabular}

Table 2. animal species and quantity. The quantities are reported according to the scheme required by the Coastal Fish Fauna Monitoring Program in the Marine Protected areas of the Ministry of the 62nvironment and the Protection of the Territory and the Sea

\begin{tabular}{|c|c|c|c|c|c|c|c|c|}
\hline \multirow{2}{*}{$\begin{array}{l}\text { Zone } \\
\text { Bathymetric } \\
\text { interval } \\
\end{array}$} & \multicolumn{4}{|c|}{ A } & \multirow{2}{*}{\multicolumn{2}{|c|}{$12-18 m$}} & & \\
\hline & \multicolumn{2}{|l|}{$4-7 \mathrm{~m}$} & & \multirow[b]{2}{*}{8} & & & & \\
\hline $\begin{array}{l}\text { Replica } \\
\text { transetto }\end{array}$ & 5 & 6 & 7 & & 9 & 10 & 11 & 12 \\
\hline \multicolumn{9}{|l|}{ Cnidaria } \\
\hline $\begin{array}{l}\text { Pelagia } \\
\text { noctiluca } \\
\text { Forsskål, } 1775\end{array}$ & & & 1 & & & & & \\
\hline \multicolumn{9}{|l|}{ Ctenophora } \\
\hline $\begin{array}{l}\text { Leucothea } \\
\text { multicornis } \\
\text { Quoy \& } \\
\text { Gaimard, } 1824\end{array}$ & 1 & & & & & & & \\
\hline \multicolumn{9}{|l|}{ Echinodermata } \\
\hline $\begin{array}{l}\text { Ophidiaster } \\
\text { ophidianus } \\
\text { Lamarck, } 1816\end{array}$ & & & & 1 & & & & \\
\hline \multicolumn{9}{|l|}{ Chordata } \\
\hline $\begin{array}{l}\text { Chromis } \\
\text { chromis } \\
\text { Linnaeus, } 1758\end{array}$ & 75 & 20 & 75 & 40 & 20 & 20 & 20 & 40 \\
\hline $\begin{array}{l}\text { Coris julis } \\
\text { Linnaeus, } 1758\end{array}$ & 5 & 3 & 2 & 1 & 2 & 3 & 2 & \\
\hline $\begin{array}{l}\text { Diplodus sargus } \\
\text { Linnaeus, } 1758\end{array}$ & & & & & & & 1 & \\
\hline $\begin{array}{l}\text { Diplodus } \\
\text { vulgaris } \\
\text { Geoffroy Saint- } \\
\text { Hilaire, } 1817\end{array}$ & 8 & 5 & & 8 & 5 & & 6 & 2 \\
\hline $\begin{array}{l}\text { Oblada } \\
\text { melanura } \\
\text { Linnaeus, } 1758\end{array}$ & 8 & 4 & 3 & & 20 & 3 & & 5 \\
\hline $\begin{array}{l}\text { Sarpa salpa } \\
\text { Linnaeus, } 1758\end{array}$ & & 8 & & 8 & 3 & & 8 & 8 \\
\hline $\begin{array}{l}\text { Serranus scriba } \\
\text { Linnaeus, } 1758\end{array}$ & & 1 & & & & & & 1 \\
\hline
\end{tabular}




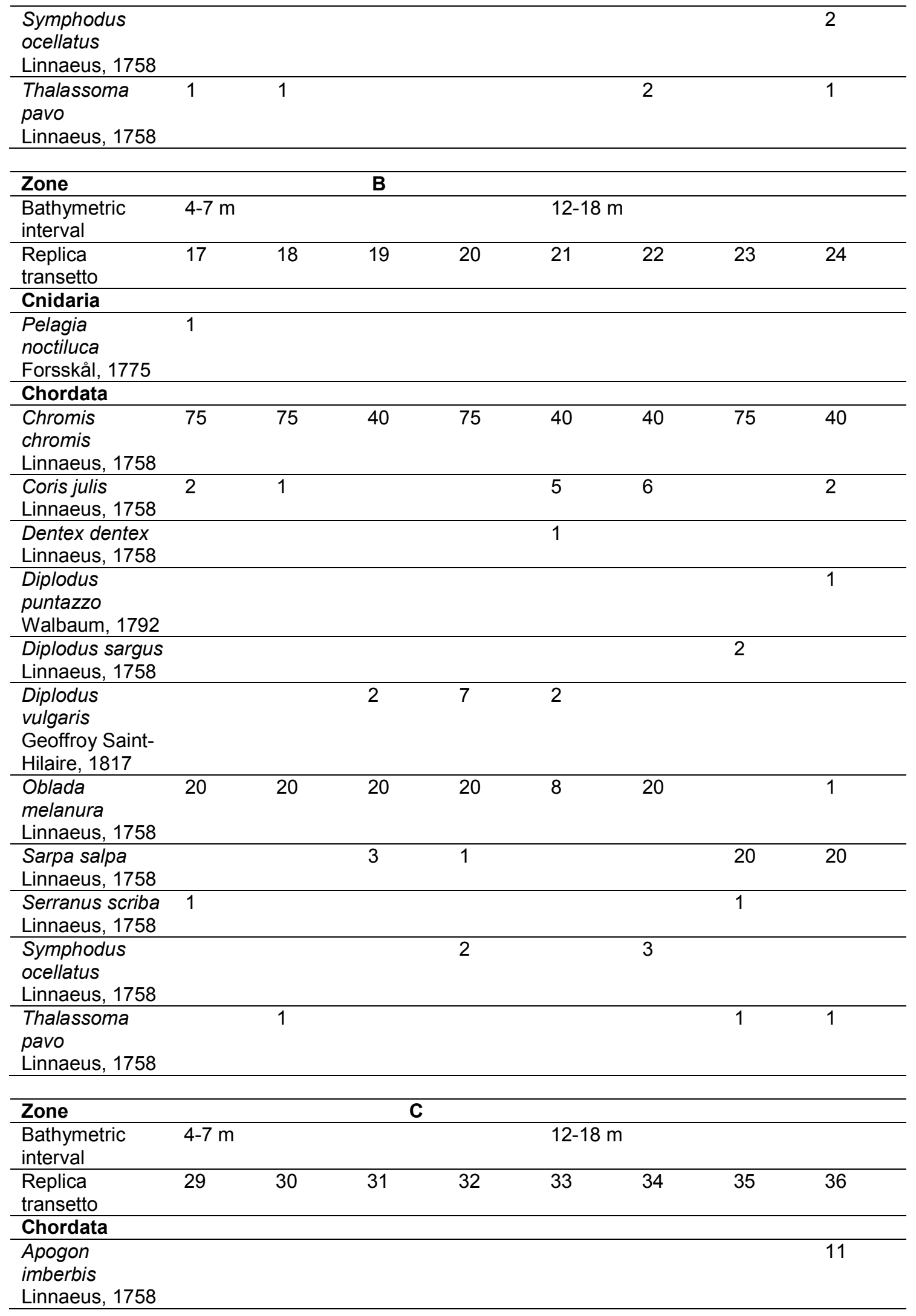


Bedini et al.; ARRB, 36(7): 58-76, 2021; Article no.ARRB.70769

\begin{tabular}{|c|c|c|c|c|c|c|c|c|}
\hline $\begin{array}{l}\text { Atherina } \\
\text { hepsetus } \\
\text { Linnaeus, } 1758\end{array}$ & & & & & 150 & & & 150 \\
\hline $\begin{array}{l}\text { Chromis } \\
\text { chromis } \\
\text { Linnaeus, } 1758\end{array}$ & 40 & 40 & 75 & 75 & 75 & 40 & 40 & 40 \\
\hline $\begin{array}{l}\text { Coris julis } \\
\text { Linnaeus, } 1758\end{array}$ & & 3 & 2 & 1 & 1 & & 2 & 1 \\
\hline $\begin{array}{l}\text { Diplodus } \\
\text { sargus } \\
\text { Linnaeus, } 1758\end{array}$ & & & & 2 & & & 1 & \\
\hline $\begin{array}{l}\text { Diplodus } \\
\text { vulgaris } \\
\text { Geoffroy Saint- } \\
\text { Hilaire, } 1817 \\
\end{array}$ & & 2 & 1 & 7 & & 1 & & 3 \\
\hline $\begin{array}{l}\text { Mullus barbatus } \\
\text { Linnaeus, } 1758\end{array}$ & & & & & & 2 & & \\
\hline $\begin{array}{l}\text { Oblada } \\
\text { melanura } \\
\text { Linnaeus, } 1758\end{array}$ & 10 & & & 20 & & & & \\
\hline $\begin{array}{l}\text { Pagellus } \\
\text { acarne } \\
\text { Risso, } 1827 \\
\end{array}$ & & & & & & 1 & & \\
\hline $\begin{array}{l}\text { Sarpa salpa } \\
\text { Linnaeus, } 1758\end{array}$ & & & & 20 & 3 & 8 & 3 & 1 \\
\hline $\begin{array}{l}\text { Serranus scriba } \\
\text { Linnaeus, } 1758\end{array}$ & 2 & 1 & & & 1 & 1 & & 1 \\
\hline $\begin{array}{l}\text { Symphodus } \\
\text { tinca } \\
\text { Linnaeus, } 1758\end{array}$ & & 1 & & & & & 1 & \\
\hline $\begin{array}{l}\text { Thalassoma } \\
\text { pavo } \\
\text { Linnaeus, } 1758\end{array}$ & 1 & & & & 2 & & 2 & 5 \\
\hline
\end{tabular}

Table 3. In the table are expressed the quantity and size of taxonomically determined species

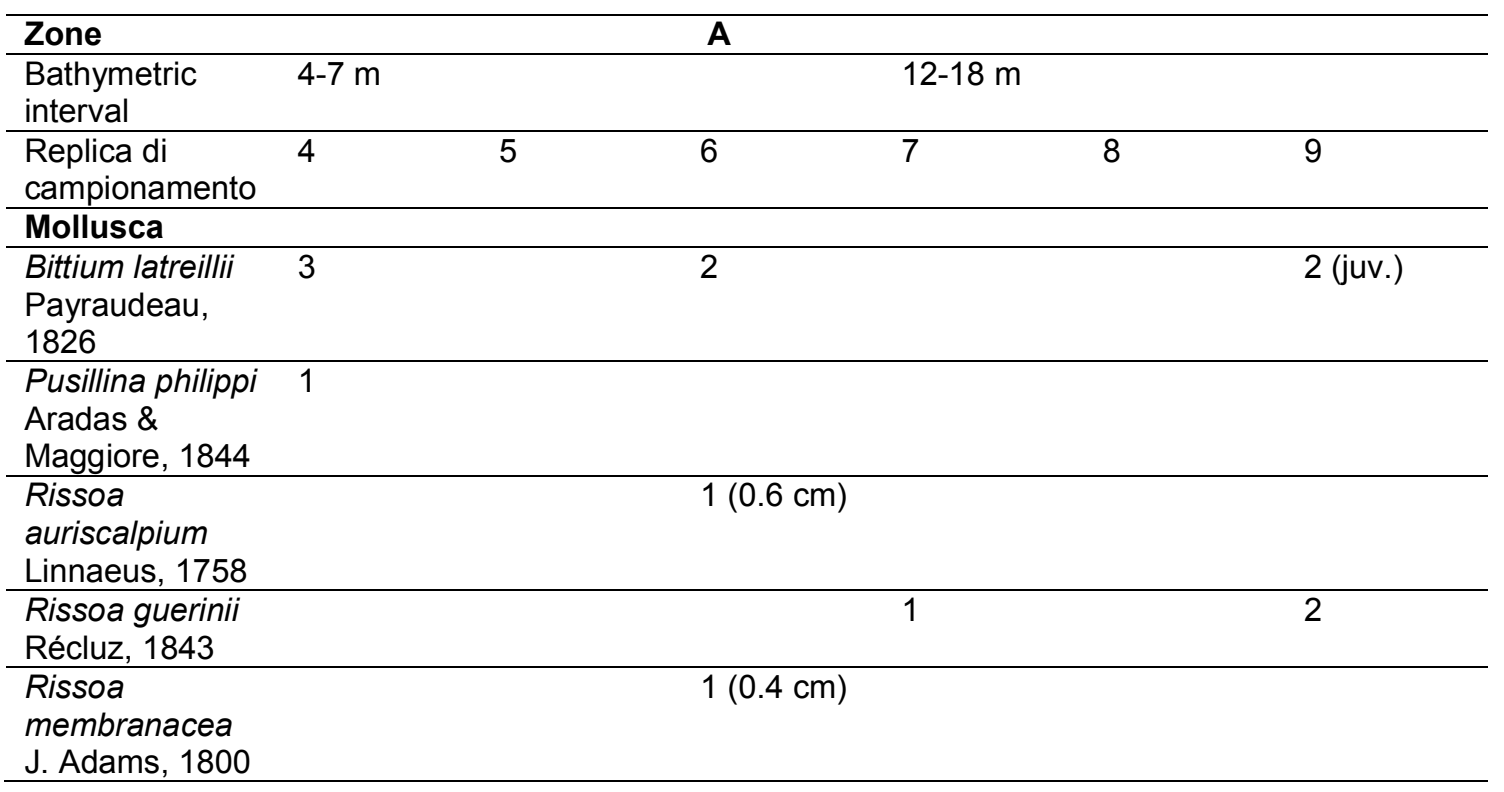




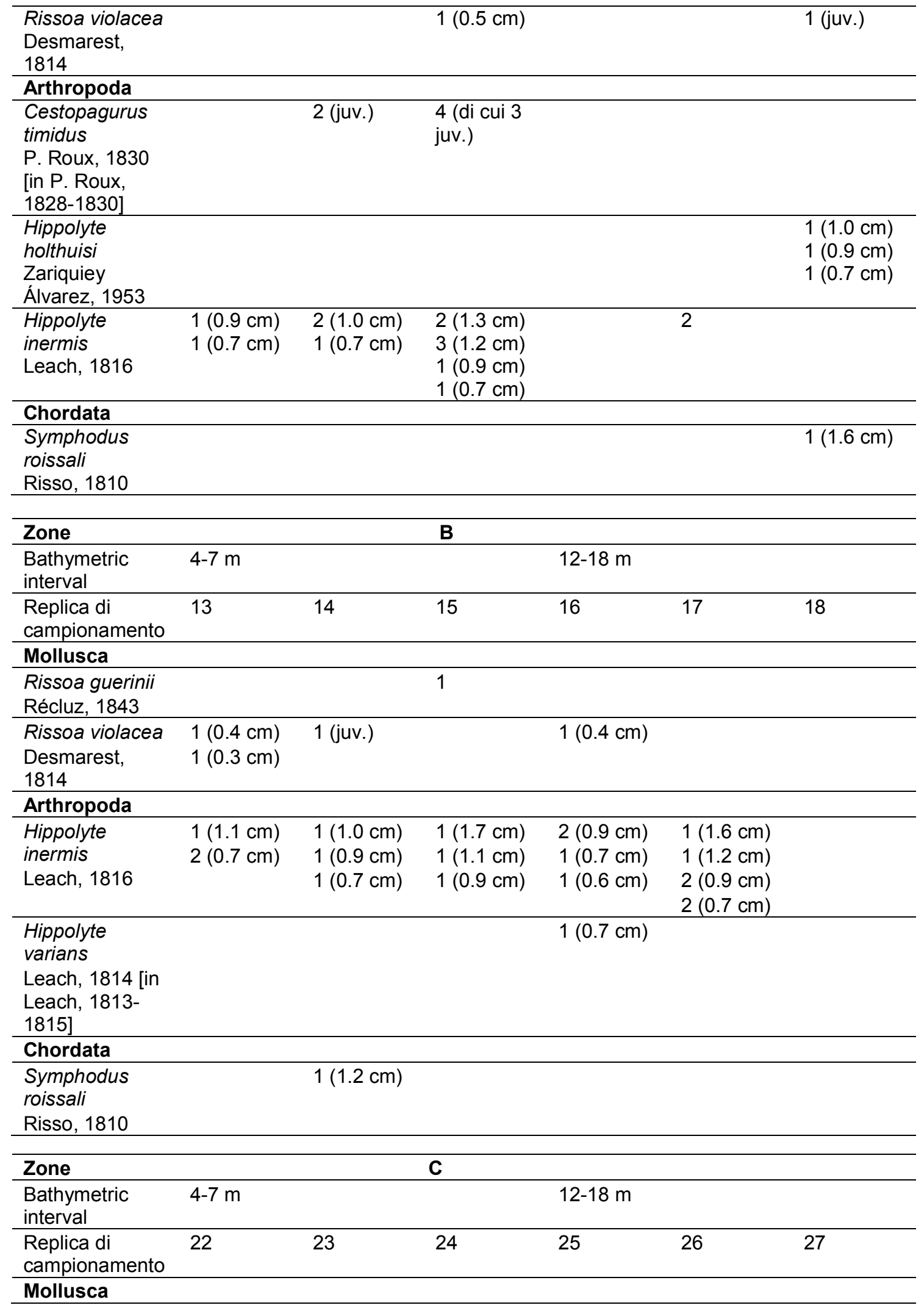




\begin{tabular}{|c|c|c|c|c|c|c|}
\hline $\begin{array}{l}\text { Aplus dorbignyi } \\
\text { Payraudeau, } \\
1826\end{array}$ & & & & & 1 & \\
\hline $\begin{array}{l}\text { Bittium } \\
\text { reticulatum } \\
\text { da Costa, } 1778\end{array}$ & & & 1 & & & \\
\hline $\begin{array}{l}\text { Cratena } \\
\text { peregrina } \\
\text { Gmelin, } 1791\end{array}$ & & & & 1 & & \\
\hline $\begin{array}{l}\text { Gibbula ardens } \\
\text { Salis } \\
\text { Marschlins, } \\
1793\end{array}$ & & & & & & 1 \\
\hline $\begin{array}{l}\text { Ocenebra } \\
\text { edwardsii } \\
\text { Payraudeau, } \\
1826\end{array}$ & & & & 1 & & \\
\hline $\begin{array}{l}\text { Pusillina } \\
\text { lineolata } \\
\text { Michaud, } 1830\end{array}$ & & & & 1 & & \\
\hline $\begin{array}{l}\text { Rissoa } \\
\text { auriscalpium } \\
\text { Linnaeus, } 1758\end{array}$ & 1 & & 1 & & 1 & \\
\hline $\begin{array}{l}\text { Rissoa } \\
\text { membranacea } \\
\text { J. Adams, } 1800\end{array}$ & 1 & & & & & \\
\hline $\begin{array}{l}\text { Rissoa violacea } \\
\text { Desmarest, } \\
1814\end{array}$ & & 1 & & & & \\
\hline \multicolumn{7}{|l|}{ Arthropoda } \\
\hline $\begin{array}{l}\text { Hippolyte } \\
\text { inermis } \\
\text { Leach, } 1816\end{array}$ & $\begin{array}{l}2(0.8 \mathrm{~cm}) \\
1(0.7 \mathrm{~cm}) \\
2(0.4 \mathrm{~cm})\end{array}$ & $\begin{array}{l}1(1.2 \mathrm{~cm}) \\
2(1.0 \mathrm{~cm}) \\
1(0.4 \mathrm{~cm})\end{array}$ & $\begin{array}{l}1(1.3 \mathrm{~cm}) \\
1(1.1 \mathrm{~cm}) \\
1(0.9 \mathrm{~cm}) \\
3(0.6 \mathrm{~cm})\end{array}$ & $\begin{array}{l}1(0.9 \mathrm{~cm}) \\
1(0.7 \mathrm{~cm})\end{array}$ & $\begin{array}{l}2(0.9 \mathrm{~cm}) \\
2(0.7 \mathrm{~cm}) \\
1(0.4 \mathrm{~cm})\end{array}$ & $\begin{array}{l}1(1.1 \mathrm{~cm}) \\
1(0.8 \mathrm{~cm}) \\
2(0.7 \mathrm{~cm})\end{array}$ \\
\hline \multicolumn{7}{|l|}{ Chordata } \\
\hline $\begin{array}{l}\text { Syngnathus } \\
\text { acus } \\
\text { Linnaeus, } 1758\end{array}$ & & $\begin{array}{l}1(8 \mathrm{~cm} ; \\
\text { juv. })\end{array}$ & & & & \\
\hline
\end{tabular}

Table 4. species taxonomically determinated by the observation of photos and videos taken in the study area

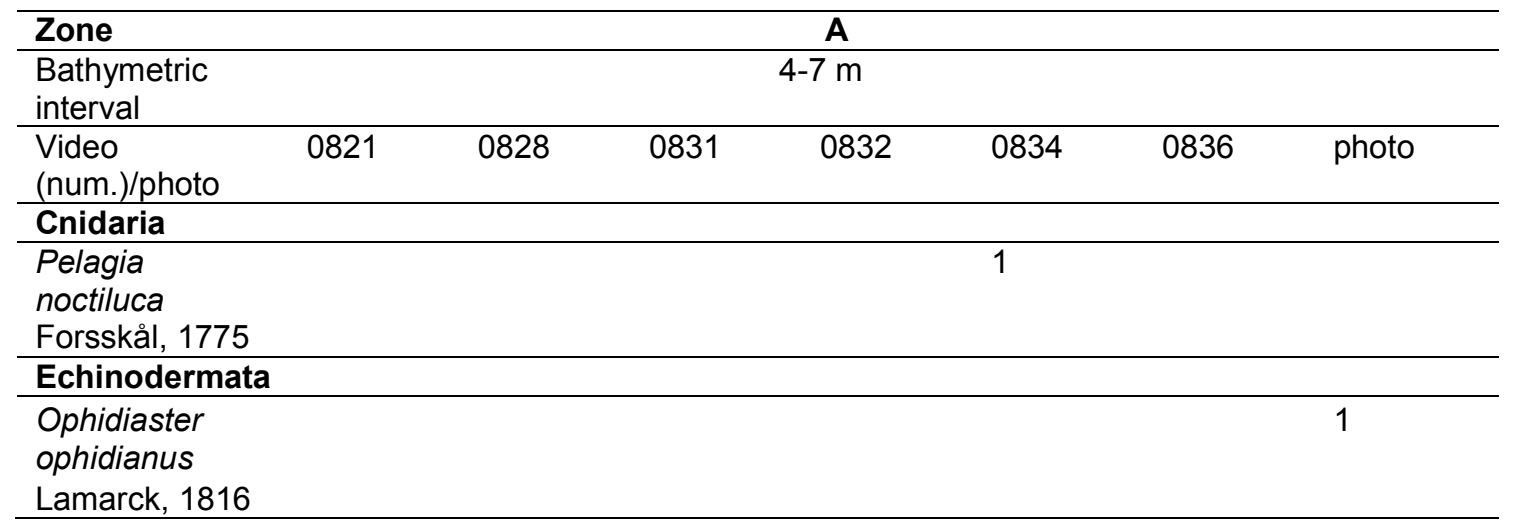




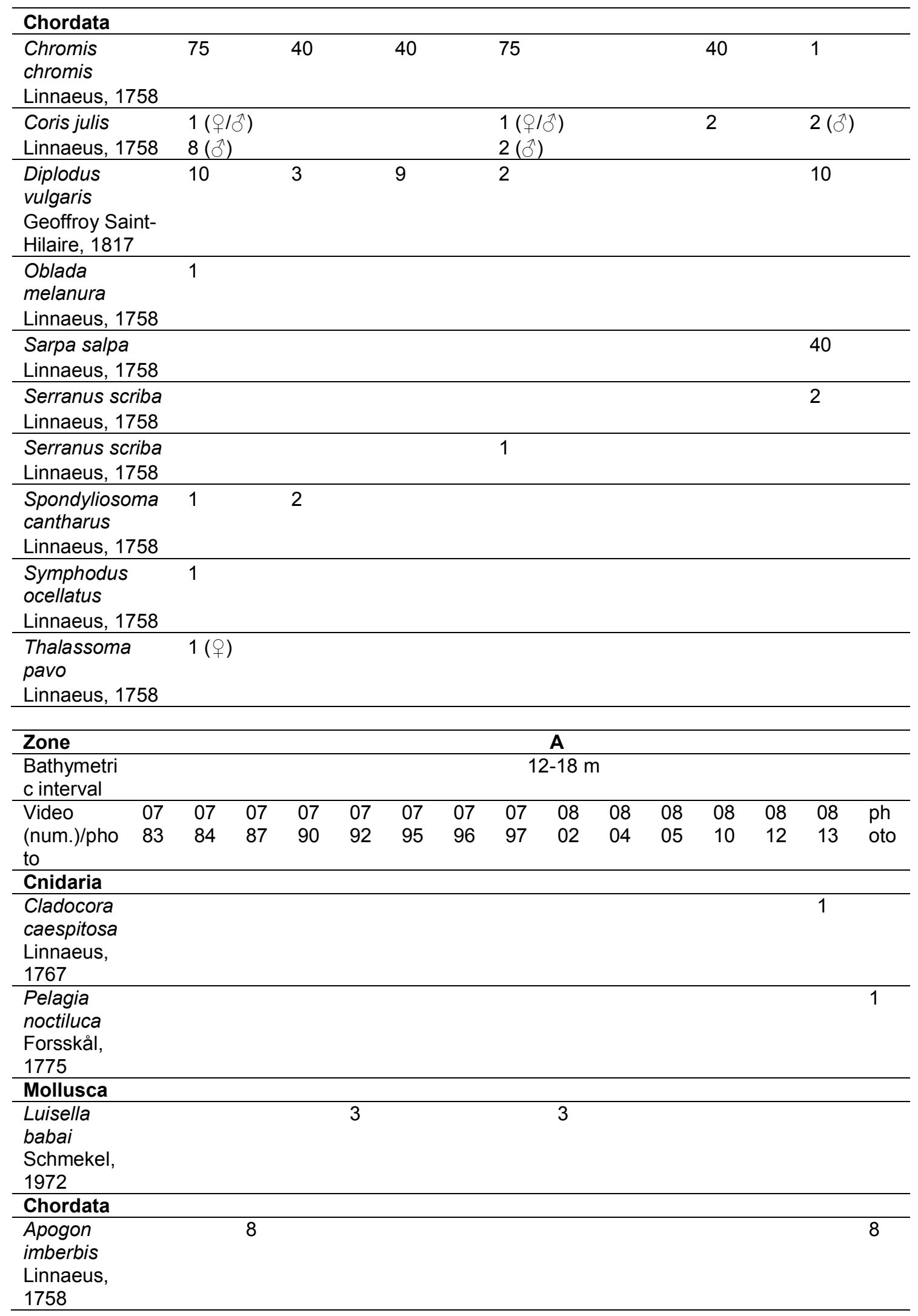




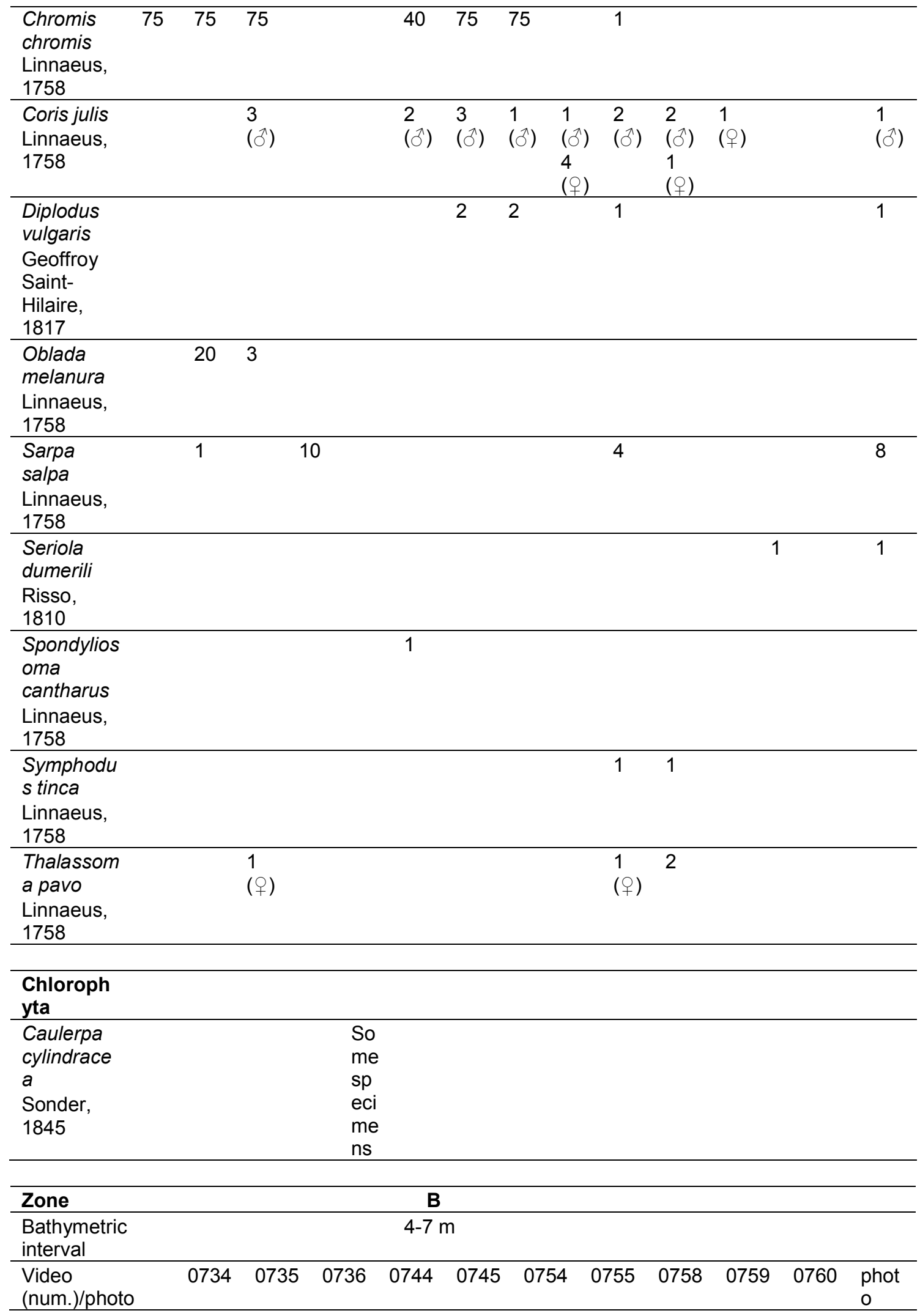


Bedini et al.; ARRB, 36(7): 58-76, 2021; Article no.ARRB.70769

\begin{tabular}{|c|c|c|c|c|c|c|c|c|c|c|c|}
\hline Chordata & & & & & & & & & & & \\
\hline $\begin{array}{l}\text { Chromis } \\
\text { chromis } \\
\text { Linnaeus, } 1758\end{array}$ & 150 & 75 & 75 & 75 & 150 & 40 & 40 & 40 & 75 & 75 & 40 \\
\hline $\begin{array}{l}\text { Coris julis } \\
\text { Linnaeus, } 1758\end{array}$ & & & $1\left(\delta^{\lambda}\right)$ & $3\left(\delta^{\lambda}\right)$ & $\begin{array}{l}2 \\
(\phi / \delta \\
1 \\
1(0)\end{array}$ & $2\left({ }^{\lambda}\right)$ & & & $\begin{array}{l}4\left(0^{\Uparrow}\right) \\
1 \\
\left(+/{ }^{\lambda}\right. \\
)^{4}\end{array}$ & $\begin{array}{l}3 \\
\left(\mathrm{P} /{ }^{\Uparrow}\right. \\
)^{4}\end{array}$ & $\begin{array}{l}2 \\
(\text { I/ठ } \\
)\end{array}$ \\
\hline $\begin{array}{l}\text { Diplodus } \\
\text { vulgaris } \\
\text { Geoffroy Saint- } \\
\text { Hilaire, } 1817\end{array}$ & & & & & & & & & & 1 & \\
\hline $\begin{array}{l}\text { Oblada } \\
\text { melanura } \\
\text { Linnaeus, } 1758\end{array}$ & & & 2 & & 1 & & & & 8 & 6 & \\
\hline $\begin{array}{l}\text { Sarpa salpa } \\
\text { Linnaeus, } 1758\end{array}$ & & & & & & & 17 & & & & 40 \\
\hline $\begin{array}{l}\text { Serranus scriba } \\
\text { Linnaeus, } 1758\end{array}$ & & & & & 1 & & & 1 & & & \\
\hline $\begin{array}{l}\text { Symphodus } \\
\text { tinca } \\
\text { Linnaeus, } 1758\end{array}$ & & & & 2 & & & & & & & \\
\hline $\begin{array}{l}\text { Thalassoma } \\
\text { pavo } \\
\text { Linnaeus, } 1758\end{array}$ & & & & $\begin{array}{l}2\left(\begin{array}{l}1 \\
0\end{array}\right) \\
2(+)\end{array}$ & & $1(\overbrace{}^{\lambda})$ & & & & & \\
\hline Zone & & & & B & & & & & & & \\
\hline $\begin{array}{l}\text { Bathymetric } \\
\text { interval }\end{array}$ & & & & $12-1$ & $8 \mathrm{~m}$ & & & & & & \\
\hline $\begin{array}{l}\text { Video } \\
\text { (num.)/photo }\end{array}$ & 0719 & & 0720 & & 072 & & & 0731 & & photo & \\
\hline Chordata & & & & & & & & & & & \\
\hline $\begin{array}{l}\text { Chromis } \\
\text { chromis } \\
\text { Linnaeus, } 1758\end{array}$ & 40 & & 75 & & 75 & & & 75 & & 75 & \\
\hline $\begin{array}{l}\text { Coris julis } \\
\text { Linnaeus, } 1758\end{array}$ & $3\left(0^{1}\right)$ & & $1\left({ }^{\Uparrow}\right)$ & & & & & & & $1\left(0^{1}\right)$ & \\
\hline $\begin{array}{l}\text { Diplodus } \\
\text { vulgaris } \\
\text { Geoffroy Saint- } \\
\text { Hilaire, } 1817 \\
\end{array}$ & & & & & 2 & & & 7 & & 1 & \\
\hline $\begin{array}{l}\text { Oblada } \\
\text { melanura } \\
\text { Linnaeus, } 1758\end{array}$ & 10 & & 2 & & 3 & & & 44 & & 9 & \\
\hline $\begin{array}{l}\text { Sarpa salpa } \\
\text { Linnaeus, } 1758\end{array}$ & & & & & & & & & & 20 & \\
\hline $\begin{array}{l}\text { Serranus scriba } \\
\text { Linnaeus, } 1758\end{array}$ & & & & & & & & & & 2 & \\
\hline $\begin{array}{l}\text { Thalassoma } \\
\text { pavo } \\
\text { Linnaeus, } 1758\end{array}$ & & & & & & & & & & & \\
\hline
\end{tabular}


Bedini et al.; ARRB, 36(7): 58-76, 2021; Article no.ARRB.70769

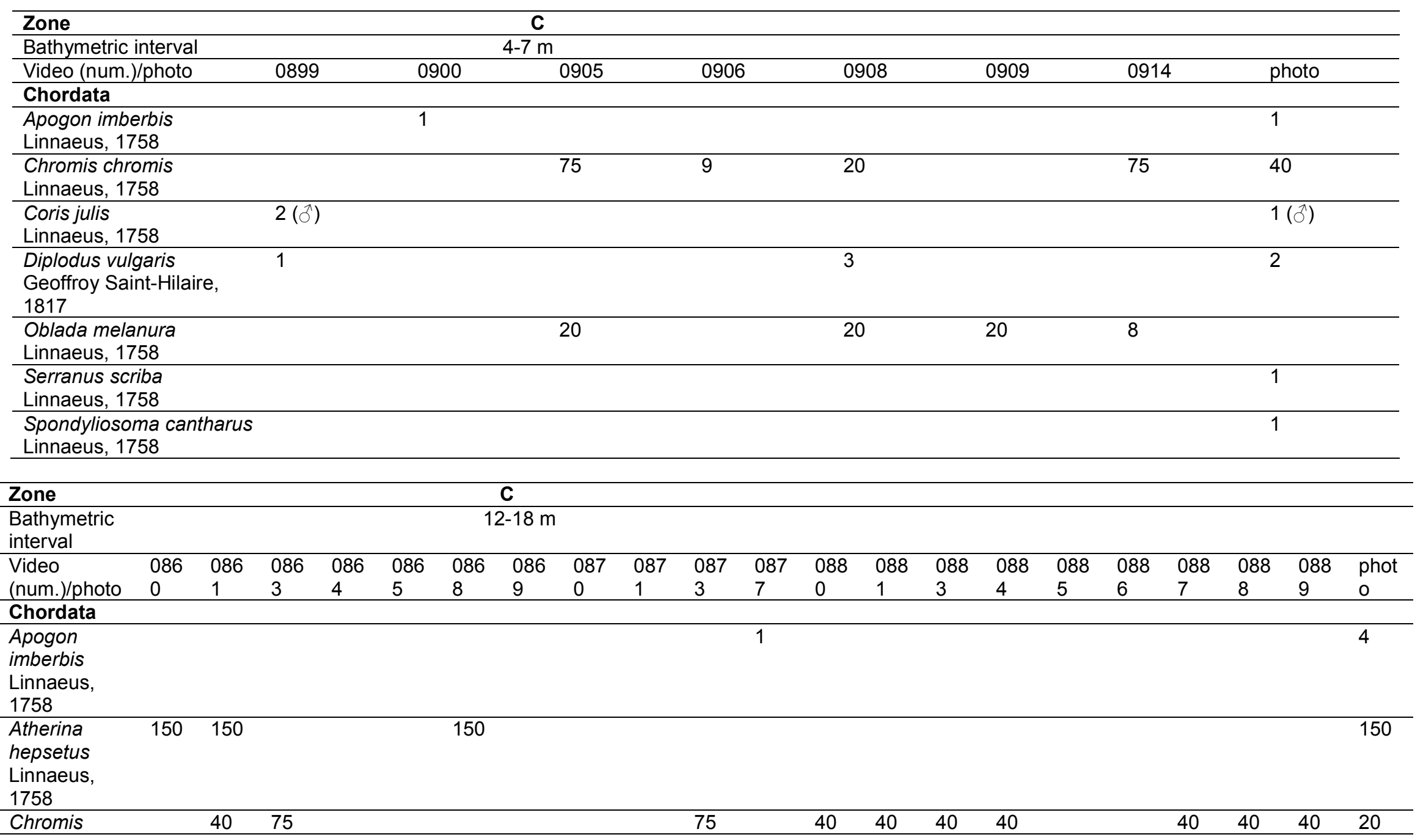


Bedini et al.; ARRB, 36(7): 58-76, 2021; Article no.ARRB.70769

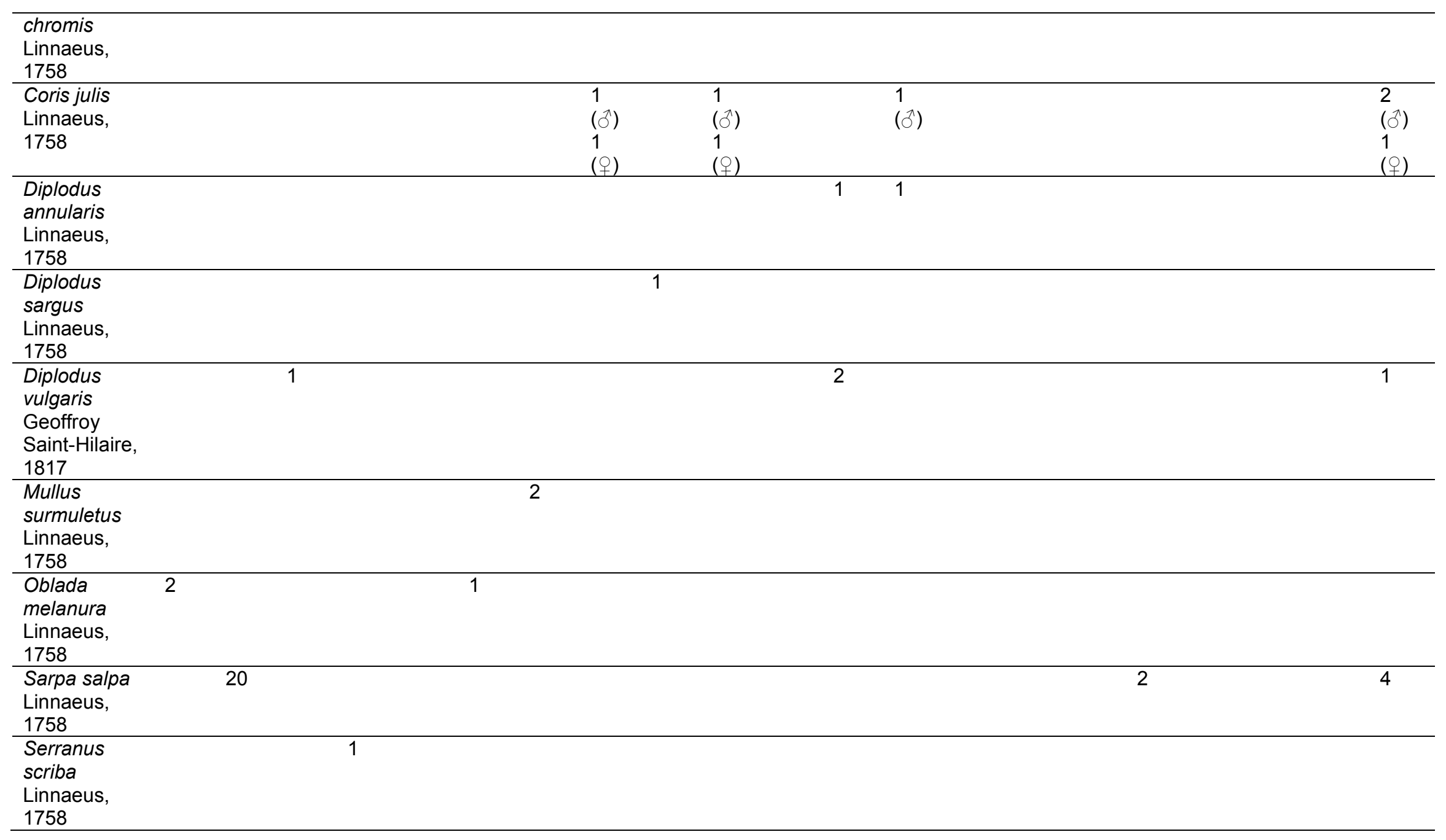


Bedini et al.; ARRB, 36(7): 58-76, 2021; Article no.ARRB.70769

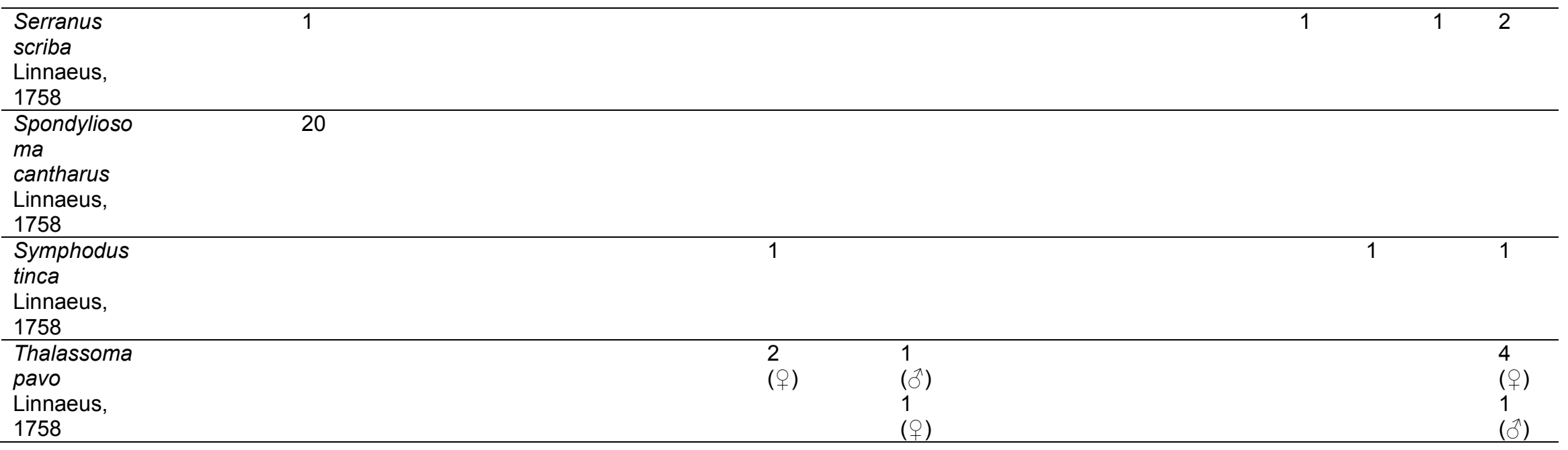




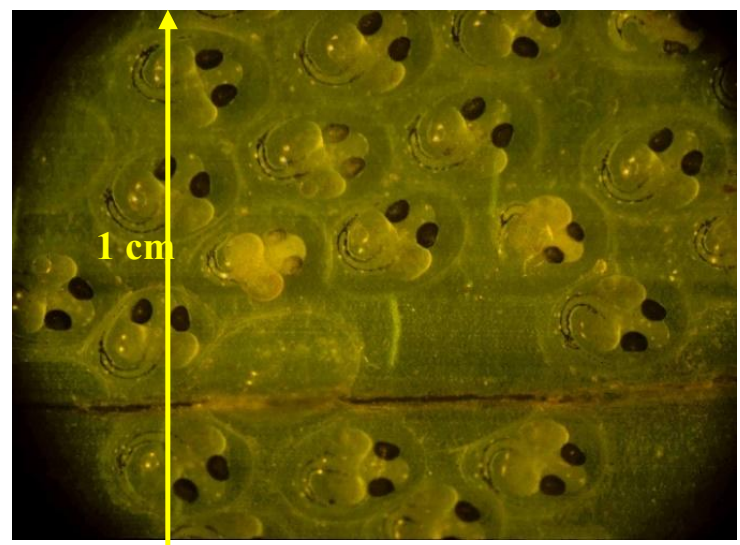

Fig. 7.

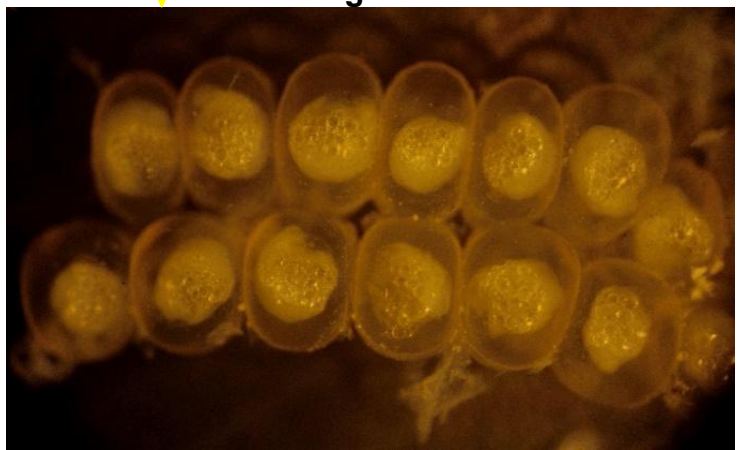

Fig. 9.

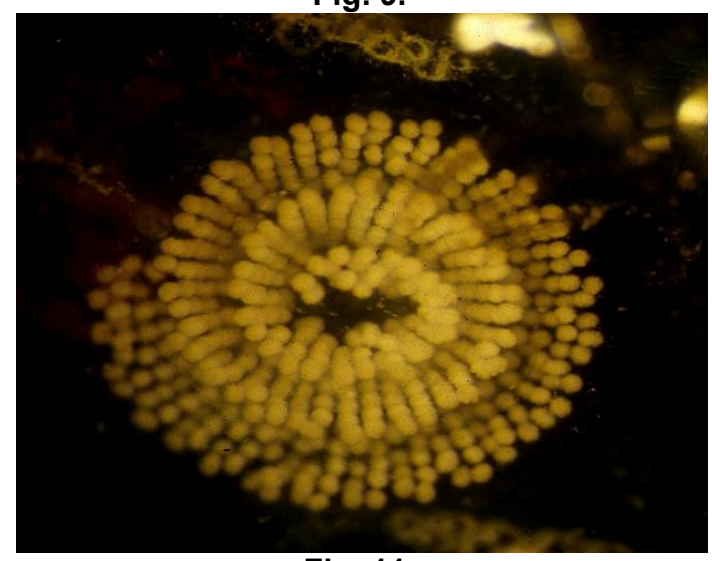

Fig. 11.

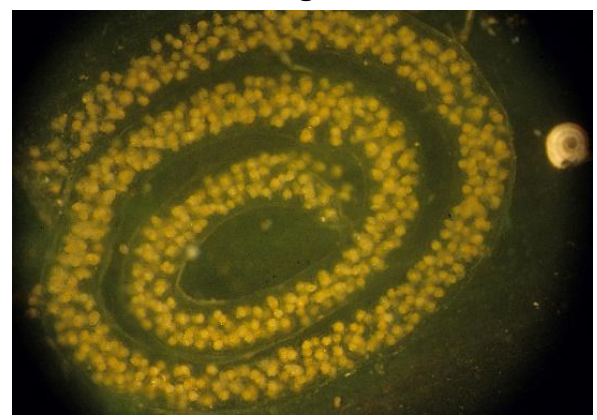

Fig. 13.

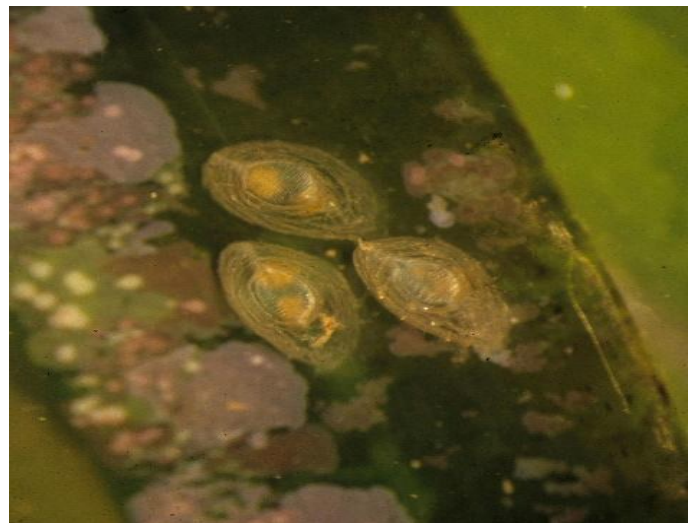

Fig. 8.

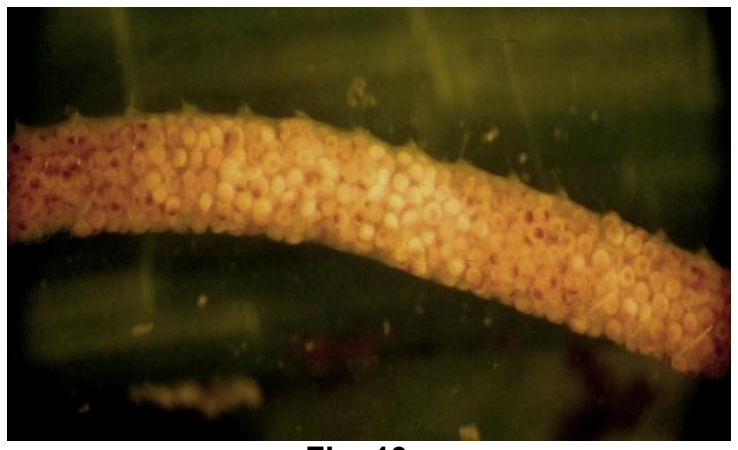

Fig. 10.

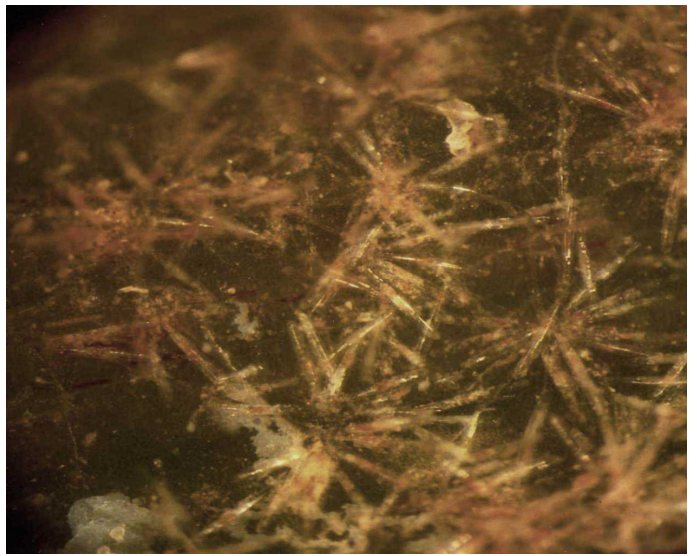

Fig. 12.

Fig. 7-13. Eggs on P. oceanica leaves 


\section{Bryozoans on P. oceanica leaves}

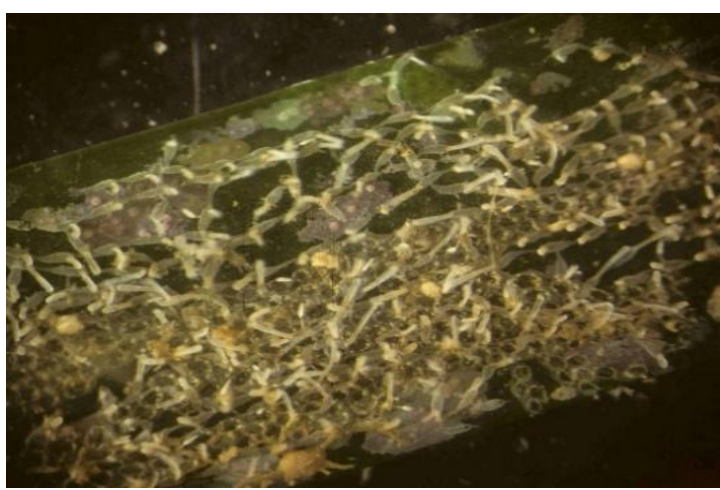

Fig. 14. Aetea lepadiformis Waters, 1906

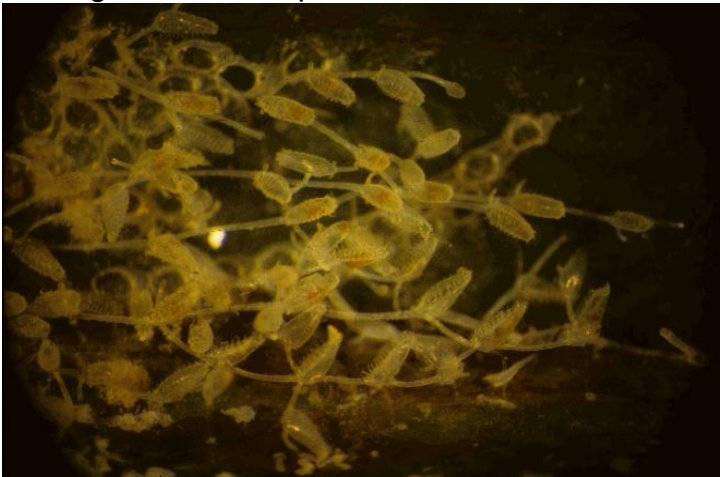

Fig. 16. Beania mirabilis Johnston, 1840

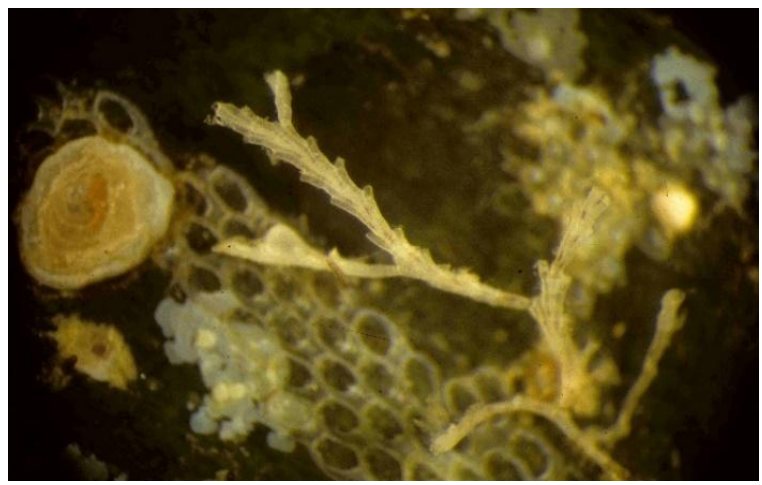

Fig. 18. Crisia ramosa Harmer, 1891

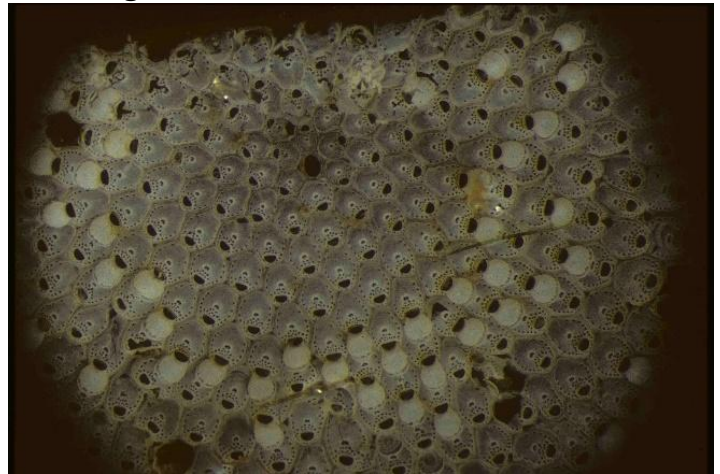

Fig. 20. Fenestrulina malusii (Audouin, 1826)

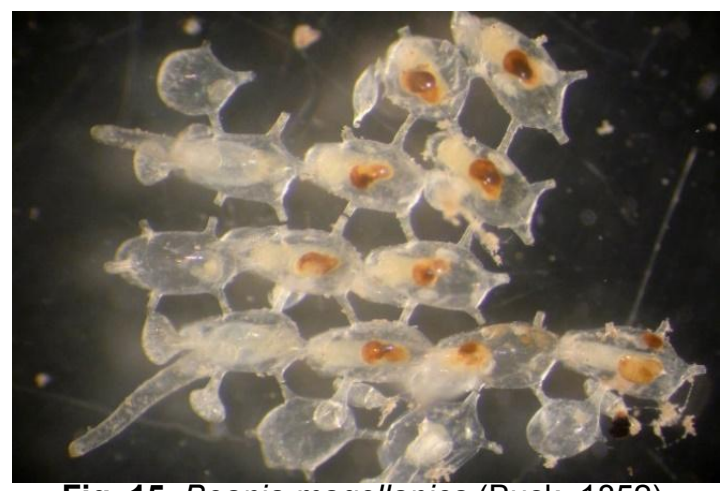

Fig. 15. Beania magellanica (Busk, 1852)

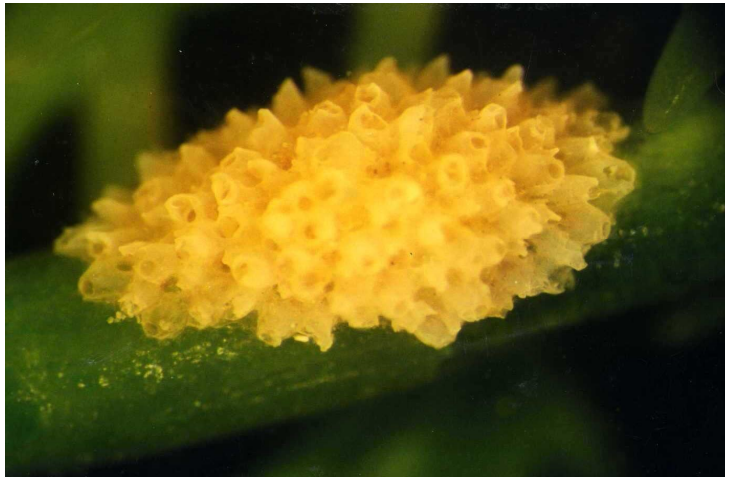

Fig. 17. Cellepora pumicosa (Pallas, 1766)

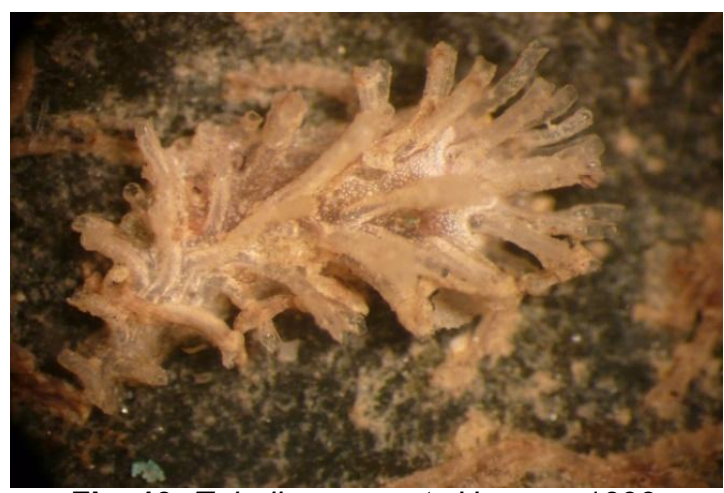

Fig. 19. Tubulipora aperta Harmer, 1898 


\section{DISCUSSION}

Study the very important nursery function of the Posidonia oceanica (L.) Delile, 1813 meadows also helps to understand the ecological situation of the entire surrounding area. In this study the peculiarity was the continuous alternation of rocks and the use of numerous and complementary sampling methods to have a real and exact evaluation of the nursery function. The results obtained allow to evaluate the nursery function of the prairie very actively in the Protected Marine Area of Ventotene and Santo Stefano.

\section{CONCLUSION}

The investigation carried out in the three zones A, B, C of the Marine Protected Areas of the islands of Ventotene and Santo Stefano have highlighted a good ecological situation of the sessile and vagile biocenoses present in the $P$. oceanica. The density of the bunches is almost everywhere very dense. Samplings with plankton nets highlighted the presence of rare juvenile specimens of Hippolytidae and Rissoidae have been captured. Particulary important for demonstration that the prairies investigated perfectly perform they nursery function was the identification, on the leaves studied in our laboratories, of many species of eggs and Bryozoans.

\section{COMPETING INTERESTS}

Authors have declared that no competing interests exist.

\section{REFERENCES}

1. Giaccone G, Sortino M. Biogeographical significance of the marine vegetation of sicily and the smaller islands of the mediterranean sea area. Studies and Information Bulletin of the Colonial Garden of Palermo Italian. 1974;26:130-146.

2. Harmelin-vivien ML, harmelin JG. Presentation d'une méthoded'évaluation " in situ " de la fauna ichtyologique. Ravaux Scientifiques Du Parc National De PortCros. 1975;1. 47-52. French.

3. Bianchi CN, Cocito S, Morri C, Sgorbini S. Underwater bionomic detection. In: abbiati $\mathrm{m}$. (ed), lessons of the training course for underwater Scientific Researcher. International School for Scientific Diving, Pisa 1991; 67-83. Italian.
4. Balduzzi A, Bianchi Cn, Cattaneo-Vietti R, Cerrano C, Cocito S, Cotta S, et al. First features of benthic bionomy of the gallinaria island (ligurian sea). Acts $x$ congr. A.i.ol. 1994;603-617.

5. Bedini R. The ecosystem in posidonia oceanica (I.) Delile as a biondicator of water quality. Proceedings "the state of the environment in tuscany" Tuscany Region Editions 1995;4:27-42. Italian.

6. Bedini R. Posidonia oceanica seed culture experiments in the aquarium. Proceedings of the tuscan society of natural sciences series b. 1997; 104: 9-15. Italian.

7. Bedini R, Channels MG. Development of posidonia oceanica seedlings in the laboratory starting from beached seeds. Sea environment. Ecology and new research technologies. Series "scientific and technological research" n. 12, tuscany region editions 1997: 85-92. Italian.

8. Bedini R, Canali MG, Baldi C. Germination and cultivation tests of posidonia oceanica (I.) Delile seeds in the laboratory. Mediterranean marine biology, 1997;4(1):356-358. Italian

9. Bedini R, Canali MG, Baldi C. Laboratory cultivation tests of posidonia oceanica (L.) Delile starting from seeds. Proceedings of the fist geoitalia conference, 5-8 september 2001, chieti. Italian.

10. Bedini R, Nannelli A, Battistini F. Restoration of posidonia oceanica (I.)Delile meadows: is there an effective methodology? Journal of life sciences, 2013;7(7): 722-726.

11. Bedini R, Bedini M, Salvadori E. A new transplanting method of posidonia oceanica plants. Firenze university press, eighth international symposium "monitoring of mediterranean coastal areas. Problems and Measurement Techniques". 2020;492500.

Doi: 10.36253/978-88-5518-147-1.4.

12. Bedini R, Canali MG, Acunto S. Study of the vagile fauna of the posidonia oceanica (I.) Delile meadows of the gulfs of follonica and baratti: decapod crustaceans and molluscs. Sea environment. Ecology and new research technologies. Series "scientific and technological research" n. 12, Tuscany Region Editions 1997;101112. Italian.

13. Bedini $\mathrm{R}$, bedini a. Mimetic adaptations and polychromaticism in the animals of the posidonia oceanica (I.) Delile prairies. Proceedings xix italian society of ethology 
conference, 4-6 october 2000, san giuliano (pisa). Italian.

14. Bedini R, Bedini M, Salvadori E. Patches, bands, blotches and changing colours is a very outstanding skill to confuse predators in some crabs living in posidonia oceanica (linnaeus) delile, 1813 meadows. Xxvii Convegno Nazionale Della Società Italiana Di Etologia, 19-21 Giugno 2017, Calci Pisa.

15. Buia MC, Gambi MC, Dappiano M. Chapter 5: seagrass systems. Biol. Mar. Medit. 2003;10(suppl.):145-198.

16. Bedini $\mathrm{R}$, canali $\mathrm{mg}$, bulleri $\mathrm{f}$, bedini $\mathrm{a}$, fantini $r$, magnarini I. Et al. Mapping of the upper limit of some posidonia oceanica meadows along the tuscan coast. Mediterranean marine biology, 2000;7(1): 499-508. Italian.

17. Morelli D, Bedini R, Magnarini L, Fantini R, Colantoni P. Extension of the posidonia oceanica (I.) Delile in some stretches of the coast of the island of elba and the gulf of follonica (northern tyrrhenian). Proceedings of the fist geoitalia conference, 5-8 september 2001, chieti. Italian 12ica meadows. Acta adriatica, 2011;52(2): 201-214.

(C) 2021 Bedini et al.; This is an Open Access article distributed under the terms of the Creative Commons Attribution License (http://creativecommons.org/licenses/by/4.0), which permits unrestricted use, distribution, and reproduction in any medium, provided the original work is properly cited.

Peer-review history:

The peer review history for this paper can be accessed here: https://www.sdiarticle4.com/review-history/70769 